\title{
Antimonene/Bismuthene Vertical Van-der Waals Heterostructure: A Computational Study
}

\author{
Shobair Mohammadi Mozvashia ${ }^{a}$, Sahar Izadi Vishkayi ${ }^{\mathrm{b}}$, Meysam Bagheri Tagani ${ }^{\mathrm{a}, *}$ \\ ${ }^{a}$ Department of physics, Computational Nanophysics Laboratory (CNL), University of Guilan, P.O.Box 41335-1914, Rasht, Iran \\ ${ }^{b}$ School of Nano Science, Institute for Research in Fundamental Sciences (IPM), P. O. Box 19395-5531, Tehran, Iran
}

\begin{abstract}
In this paper, the structural, electronic, mechanical and optical properties of Antimonene/Bismuthene Van-der Waals heterostructure ( $\mathrm{Sb} / \mathrm{Bi} \mathrm{HS}$ ) were calculated based on the first principle density functional theory. We explored different stacks of Sb/Bi HS to find the most, and the least stable staking for this heterostructure. At the GGA level of theory, the most stable model is a semiconductor with an indirect bandgap of $159 \mathrm{meV}$. However, when the spin-orbit (SO) interaction is considered, the VBM and CBM touch the Fermi level and the HS becomes a semimetal. Our results also show that the electronic properties of the HS are robust against the external electric field and biaxial strain. Young's modulus was calculated of $64.3 \mathrm{~N} / \mathrm{m}$ which predicts this HS as a resistant material against being stretched or compressed. The calculated optical properties, similar to monolayer Antimonene, are completely dependent on the polarization of incident light and differ when parallel or perpendicular polarization is considered. Moreover, the absorption coefficient of $\mathrm{Sb} / \mathrm{Bi} \mathrm{HS}$ for perpendicular polarization in the visible region is significantly increased in comparison with the monolayer Antimonene. High structural stability, electronic and mechanical robustness against electric field and strain, along with polarization-dependent optical properties of this HS, promise for its applications in beam splitters and nano-scale mirrors.
\end{abstract}

Keywords: DFT, 2D materials, Antimonene, Bismuthene, Heterostructure

\section{Introduction}

Two dimensional (2D) materials, due to their unique properties, such as high carrier mobility [1], superior mechanical characteristics [2], and high ratio between their lateral size (1-1000 $\mu \mathrm{m})$ and thickness $(<1 \mathrm{~nm})[3]$ have attracted a great deal of attention in the last decade, and annually this attraction becomes even greater. The discovery of Graphene and its unique properties in 2004 [4], opened a road to new investigations and observations in 2D materials because, before that, it assumed that 2D materials are thermodynamically unstable [5]. After that, several types of 2D materials theoretically investigated, and/or experimentally exfoliated, including hexagonal boron nitride, Transition metal dichalcogenides, perovskites, and other elemental 2D materials such as group IV and V monolayers [613]. Because of various applications, such as sensors [14], field effect transistors [15], light-emitting diodes [16], solar cells [17], and energy-conserving devices [18] in nano-scale size, the research interest in 2D materials remains attractive for many years.

Antimonene and Bismuthene, from group $\mathrm{V}$ elemental monolayers, are relatively novel members of 2D materials which their electronic, mechanical and optical properties have been investigated in numerous experimental and theoretical studies in recent years [19-25]. The studies imply that these two monolayers are both stable in $\beta$ (buckled hexagonal) phase and predict an indirect bandgap for Antimonene and a direct narrow

\footnotetext{
${ }^{*}$ Corresponding author

Email address: m_bagheri@guilan.ac.ir (Meysam Bagheri Tagani)
}

bandgap for Bismuthene. Besides, it is predicted that the electronic properties of Antimonene are sensitive to strain and it is possible to tune its bandgap in this way. Wang et al. predicted that by applying a tensile biaxial strain of $5 \%$ the bandgap of Antimonene becomes direct and increases to its maximum value [19].

On the other hand, nowadays designing and investigating the Van-der Waals heterostructures has turned into an interesting method for broadening the scope of research in the field of 2D materials. Scientists can simply predict and/or observe very interesting properties by combining already discovered materials, not discovering new ones. For instance, $\mathrm{Lu}$ et al. by designing heterostructures of Antimonene and three 2D materials (Graphene, Arsenene, and h-BN), could predict heterostructures with tunable bandgaps between 0 to $1 \mathrm{eV}$ which have potential applications in near and mid-infrared detectors [26]. Meng et al. reported that one can enhance the efficiency of $\mathrm{MoS}_{2}$ based solar cells, by $5.23 \%$, using a $\mathrm{MoS}_{2} / \mathrm{Si}$ heterostructure [27]. Also Chen et al. predicted the opening of a direct and tunable bandgap of $400 \mathrm{meV}$ in Germanene, by designing an Antimonene/Germanene Van-der Waals heterostructure [28].

Our purpose in this study is to apply a tensile strain of 5\% to the Antimonene, by designing an Antimonene/Bismuthene Van-der Waals heterostructure. One of the natural methods for applying a biaxial strain is to form a material on a substrate with a different lattice constant. If the substrate lattice constant is larger or smaller than the superstrate, a tensile or compressive strain applies to the latter, respectively. Reviewing the literature, it raises that the lattice constant for Bismuthene is $\sim 5 \%$ larger than Antimonene's. Therefore, by the case of forming 
an $\mathrm{Sb} / \mathrm{Bi} \mathrm{HS}$, with fixed Bismuthene substrate, a tensile strain of about $5 \%$ would be applied to the Antimonene superstrate. In this paper, we intend to probe if it is theoretically possible to design a stable Van-der Waals heterostructure with such mismatching monolayers. After investigating the structural characteristics and testing the stability, we look into the electronic, mechanical and optical properties of $\mathrm{Sb} / \mathrm{Bi} \mathrm{HS}$ and discuss its potential applications.

\section{Computational Details}

For all the simulations, the Spanish package solution, SIESTA [29] was employed which is based on self-consistent density functional theory (DFT) and standard pseudopotentials. The exchange-correlation interactions were estimated through generalized gradient approximation (GGA), with parametrization of Perdew, Burke, and Ernzerhof (PBE) [30]. For the electronic and optical properties of the heterostructures, the spinorbit coupling (SOC) was also considered in addition to GGA (SOGGA). In all the calculations, the reciprocal space was sampled by a mesh of $31 \times 31 \times 1 \mathrm{k}$ points in the Brillouin zone and the density mesh cut-off was set on $100 \mathrm{Ha}$. For considering the Van-der Waals interactions between layers, the DFT-D2 correction of Grimme [31] was used. A vacuum space of $20 \AA$ was considered in the $\mathrm{z}$-direction to prevent unwanted interactions. Besides, all the pristine structures were relaxed to a force and stress of $0.001 \mathrm{eV} / \AA$ and $0.001 \mathrm{GPa}$, respectively, but in the strain related investigations the minimization of stress was canceled.

\section{Results and Discussion}

\subsection{Monolayer Antimonene and Bismuthene}

Our quest in this part is not for expressing a novel and independent assessment about these monolayers, but only for validating our methods, and verifying prior studies. However, before bringing up the discussion about $\mathrm{Sb} / \mathrm{Bi} \mathrm{HS}$, it is needed to investigate and review the properties of each monolayer separately. The pristine structures of Antimonene and Bismuthene are buckled hexagonal, where the atoms are seen like a hexagon from the top view, but they are not in the same plane plane, that is, the bonding atoms have a distance with each other in the $\mathrm{z}$ direction, called "buckling" (Fig.1). Previous studies have calculated the lattice constant from 3.94 to $4.12 \AA$ for Antimonene $[7,19,20]$ and 4.30 to $4.39 \AA$ for Bismuthene [32-34]. We calculated the Antimonene and Bismuthene lattice constants as 4.06 and $4.26 \AA$, respectively. Regarding the variety of approximations, basis sets, pseudopotentials and code packages, this order of difference in results is inevitable, thus we assess our calculations rational and reliable.

The structures were relaxed and their cohesive energies were calculated with the equation below,

$$
E_{c}=\frac{E_{\text {sheet }}-2 E_{\text {atom }}}{2}
$$

where, $E_{\text {sheet }}$ is the total energy of each monolayer, and $E_{\text {atom }}$ is the energy of each isolated $\mathrm{Sb}$ or $\mathrm{Bi}$ atoms considering spin
Table 1: Structural parameters of monolayer Bismuthene and Antimonene. lattice constants (a), atomic bonds (R), buckilng heights (h), angles between two bonds $(\theta)$, cohesive energies $\left(E_{c}\right)$, and bandgaps $\left(E_{g}\right)$ are shown and compared with previous studies.

\begin{tabular}{lcccccc}
\hline \hline & $\mathrm{a}(\AA)$ & $\mathrm{R}(\AA)$ & $\mathrm{h}(\AA)$ & $\theta\left(^{\circ}\right)$ & $E_{c}(\mathrm{eV} /$ atom $)$ & $E_{g}, \mathrm{GGA}(\mathrm{eV})$ \\
\hline Sb & & & & & & \\
This study & 4.06 & 2.88 & 1.68 & 89.6 & -3.14 & 1.24 (ind) \\
{$[20]$} & 4.04 & 2.87 & 1.67 & 89.0 & $-2.87^{\mathrm{a}}$ & 1.04 (ind) \\
{$[19]$} & 4.12 & 2.89 & 1.64 & 90.8 & -4.03 & 0.76 (ind) \\
{$[35]$} & 4.08 & 2.88 & 1.66 & 90 & $\mathrm{n} / \mathrm{a}$ & $\mathrm{n} / \mathrm{a}$ \\
\hline $\mathbf{B i}$ & & & & & & \\
This study & 4.26 & 3.03 & 1.76 & 89.5 & -3.00 & $0.75(\mathrm{dir})$ \\
{$[32]$} & 4.30 & 3.03 & 1.75 & 90.2 & $\mathrm{n} / \mathrm{a}$ & $0.60(\mathrm{dir})$ \\
{$[33]$} & 4.34 & $\mathrm{n} / \mathrm{a}$ & 1.73 & $\mathrm{n} / \mathrm{a}$ & $\mathrm{n} / \mathrm{a}$ & $0.99(\mathrm{dir})$ \\
{$[34]$} & 4.39 & 3.07 & 1.73 & 91.2 & $-3.04^{\mathrm{a}}$ & $0.46(\mathrm{dir})^{\mathrm{b}}$ \\
\hline \hline
\end{tabular}

a A conversion of unit or sign was required. ${ }^{\mathrm{b}} \mathrm{GGA}+\mathrm{SOC}$

polarization. Based on the equation (1), the value for cohesive energy should be negative for stable structures. Generally, our results about lattice constants, bond lengths, bond angles, buckling heights, cohesive energies and bandgaps are shown and compared with previous studies in Table 1, which are in a good agreement with the literature.

Fig. 2 represents the band structures and partial density of states related to both monolayers in the pristine configurations. The band structures show a direct bandgap of $847 \mathrm{meV}$ for Bismuthene and an indirect bandgap of $1.24 \mathrm{eV}$ for Antimonene. The density of states represent that in both monolayers, the $p$ orbitals are most responsible for electronic properties, and the $\mathrm{s}$ and d orbitals do not have any significant contribution.

As mentioned above, previous studies have noted that electronic properties of Antimonene are sensitive to strain and an indirect to direct transition would occur under the tensile biaxial strain of $5 \%[19,36]$. Because our purpose in this study is to probe the behavior of Antimonene under substrate generated strain, we also investigated the effect of strain to have a comprehensive review, and re-test our computational method. The biaxial strain is defined by the equation below,

$$
\epsilon=\frac{\left(a-a_{0}\right)}{a_{0}}
$$

where $a_{0}$ and $a$ are lattice constants in pristine and strained structures, respectively. Our results show that with short biaxial tensile strain $(<4 \%)$ the bandgap of Antimonene increases. It reaches to the maximum value of $1.58 \mathrm{eV}$ under the strain of $4 \%$, and an indirect to direct transition occurs at this point. After that the bandgap decreases softly and finally under the strain of $14 \%$ it is closed and the material turns into a semimetal (Figs. 3 and 4). The behavior we predict agrees with the previous reports. Good agreement of our results about the monolayers with the literature, especially the prediction of the indirect to direct transition, makes us to trust our method and gain sufficient motivation to continue our investigations.

\subsection{Structural Properties of Antimonene/Bismuthene Heterostruc- ture}

As mentioned above, the lattice constants for Antimonene and Bismuthene are 4.06 and $4.26 \AA$, respectively. Therefore, these two monolayers have a mismatch of $4.93 \%$ with each 
(a)
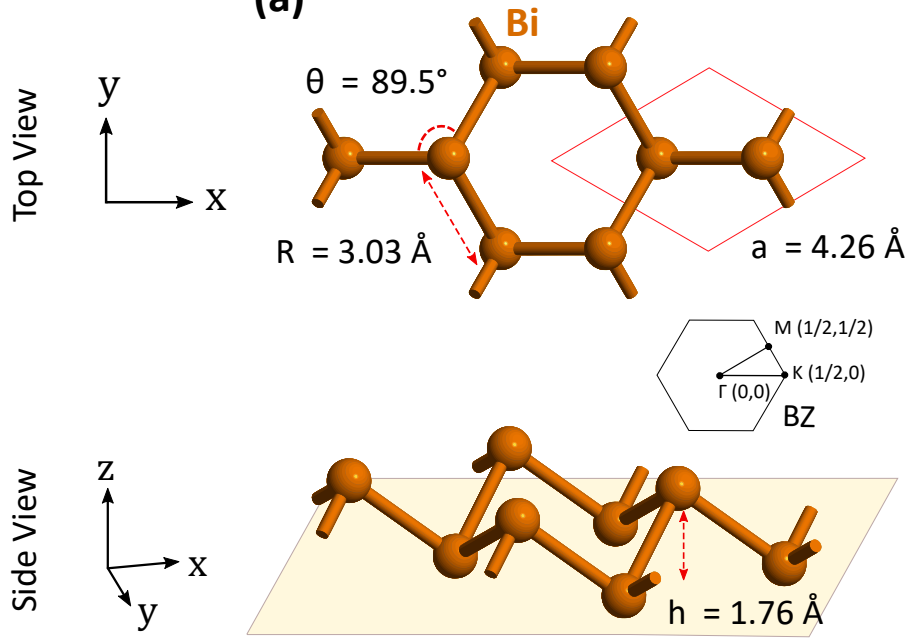

(b)

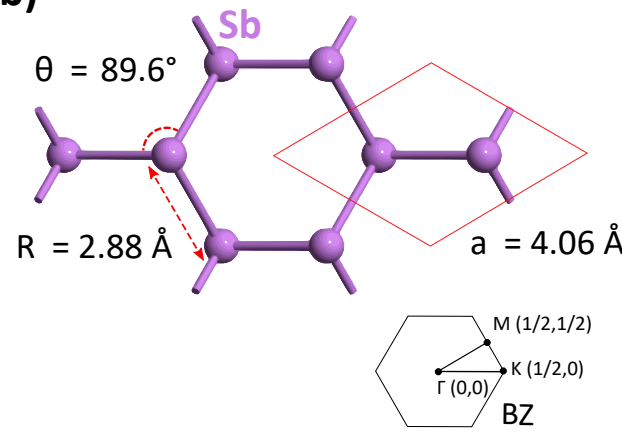

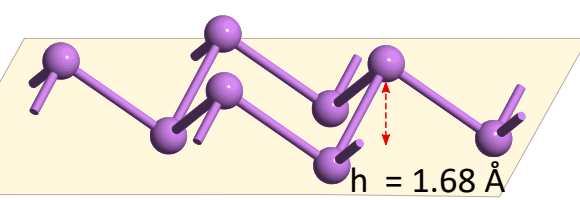

Figure 1: Structural configurations of monolayer Bismuthene (a), and Antimonene (b). unit cells, atomic bonds and Brillouin zones are depicted in the figure.

(a)

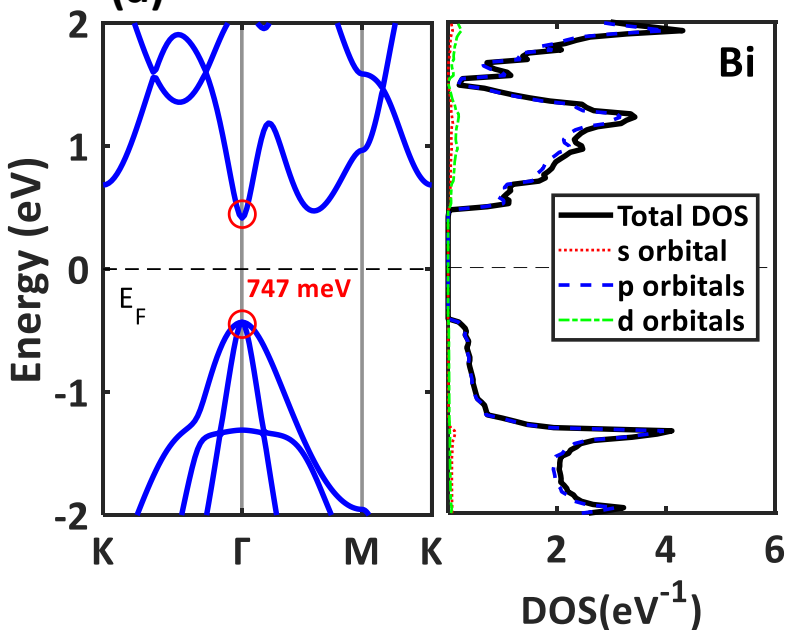

(b)

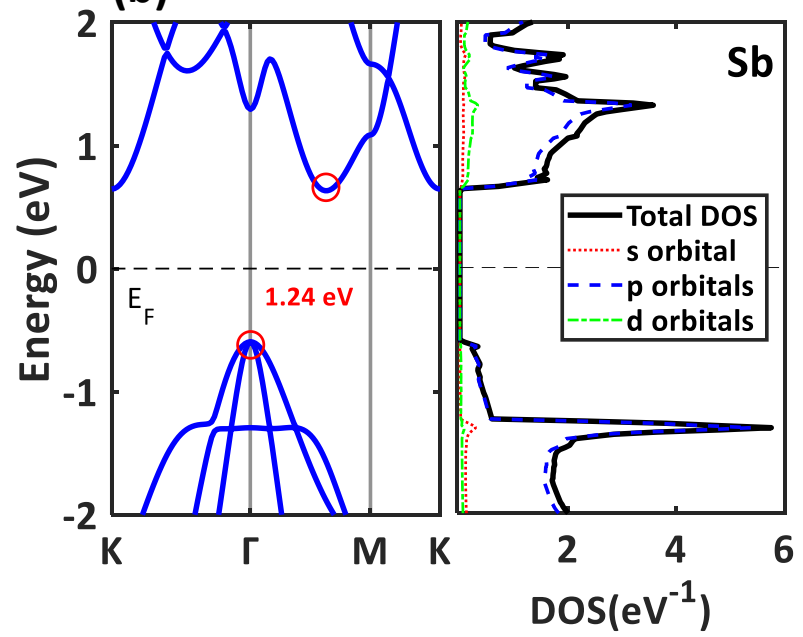

Figure 2: Band structures and density of states of Bismuthene (a), and Antimonene (b). Fermi level is shifted to zero.

other. In this study, we considered the Bi layer as a fixed substrate and let the Sb layer to relax on it. Therefore a tensile strain of $4.93 \%$ applies to the latter. We predicted four possible models for the HS. AAi, AAii, ABi, and ABii. The AAi and $\mathrm{ABi}$ models are the regular $\mathrm{AA}$ and $\mathrm{AB}$ stackings, while in AAii and ABii models the upper and lower layers have different wrinkling directions. For example in the AAi model, the upper $\mathrm{Sb}$ atom is on top of the upper $\mathrm{Bi}$ atom, while in the AAii model, the upper $\mathrm{Sb}$ atom is on top of the lower $\mathrm{Bi}$ atom. These four types of stackings are declared in Fig.5a,b. In all these models, the lattice constant is $4.26 \AA$ which is equal to monolayer Bismuthene's.

For all the models, the Sb-Sb and Bi-Bi bonds are $~ 2.94$ and $3.03 \AA$, respectively, therefore the bond lengths are independent of stacking and there is a little recombination between different models. The phonon dispersions (Fig.5c) show that three of the stackings are vibrationally stable, except Model AAii in which the imaginary modes near $\Gamma$ point show its instability. It should be noted that For calculating the cohesive energies between layers, we used the equation below,

$$
E_{c}=\frac{E_{H S}-\left(E_{S b}+E_{B i}\right)}{4}
$$

where, $E_{H S}$ is the energy of the heterostructure, and $E_{S b}, E_{B i}$ are energies of $\mathrm{Sb}$ and $\mathrm{Bi}$ layers, respectively. The largest and the smallest absolute value of cohesive energy belongs to $\mathrm{ABi}$ (-175 meV/atom) and AAii (-112 meV/atom) models, respectively, therefore, in terms of cohesive Energy, the ABi and AAii stackings are the most and the least stable models. According to Table 2, the shortest inter-layer distance (d) belongs to the ABi $(2.48 \AA)$ and the longest one is for AAii (3.92 $\AA$ ) models, respectively. The shorter inter-layer distance means that the interactions and forces are more intense between the layers and they tend to attract each other more. Therefore the ABi model turns out more favorable in this case too. Also, 

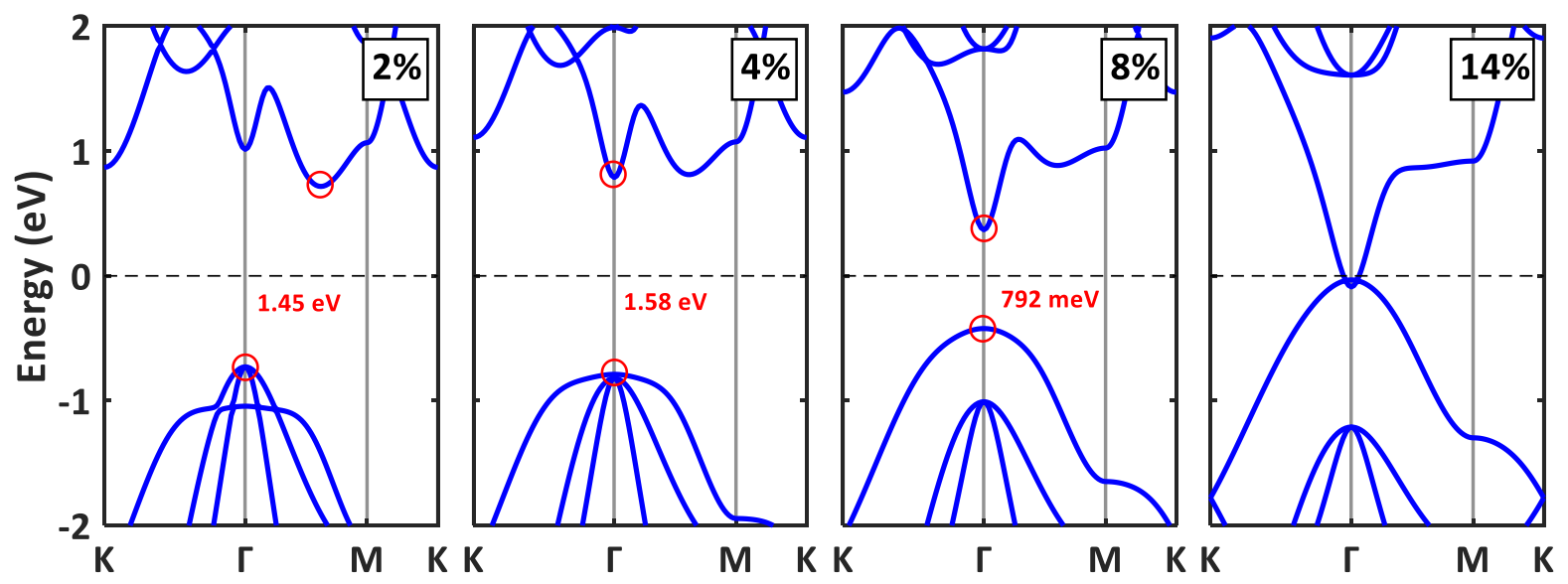

Figure 3: Variation of the GGA band structure of Antimonene with tensile strain. Each sub-figure is related to the tensile strain depicted at up-right text boxes.

Table 2: Structural parameters of four possible models of the $\mathrm{Sb} / \mathrm{Bi} \mathrm{HS}$ : $\mathrm{Sb}-\mathrm{Sb}$ and Bi-Bi bond lengths, inter-layer distances $(\mathrm{d})$, cohesive energies $\left(E_{c}\right)$, stress $(\sigma)$, and GGA bandgaps $\left(E_{g}\right)$.

\begin{tabular}{lcccccc}
\hline \hline Model & Sb-Sb $(\AA)$ & Bi-Bi $(\AA)$ & $\mathrm{d}(\AA)$ & $E_{c}(\mathrm{meV} /$ atom $)$ & $\sigma(\mathrm{N} / \mathrm{m})$ & $E_{g}(\mathrm{eV})$ \\
\hline AAi & 2.94 & 3.03 & 2.98 & -155 & 1.81 & 356 (ind) \\
AAii & 2.94 & 3.03 & 3.92 & -112 & 2.30 & 390 (dir) \\
ABi & 2.93 & 3.03 & 2.48 & -175 & 0.28 & 159 (ind) \\
ABii & 2.94 & 3.03 & 2.69 & -173 & 1.29 & 252 (ind) \\
\hline \hline
\end{tabular}

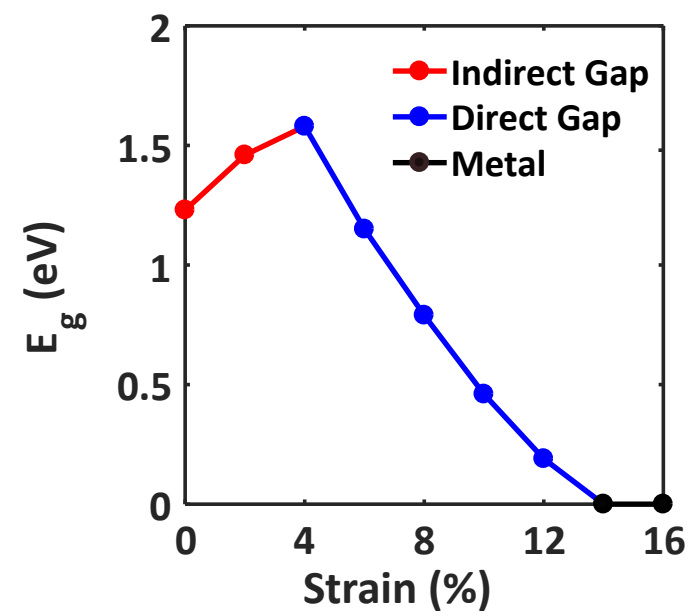

Figure 4: Variation of monolayer Antimonene GGA bandgap with tensile strain.

surface stress in ABi model is much smaller in contrast with other models, while it is the highest in model AAii. In summary, it could be said that the most stable model is $\mathrm{ABi}$, while $\mathrm{AAi}$ and ABii models are less stable and AAii model is completely unstable. Our calculations in phonon dispersion, cohesive energy, inter-layer distance, and stress among these four models are supportive of each other. By reviewing the previous works, it raises that the model we call $\mathrm{ABi}$ was usually more stable in other Antimonene based heterostructures as well. For example, Chen et al reported that in Antimonene/Germanene heterostructure, a so-called ABI model is the most stable stack- ing with a $-283 \mathrm{meV} /$ atom of cohesive energy [28]. Also, in $\mathrm{Sb} / \mathrm{InSe}$ heterostructure, the most stable model is reported a socalled H3 model with a cohesive energy of $-23.86 \mathrm{eV} / \AA^{2}$ (by multiplying by the unit cell area and dividing into the number of atoms, the converted cohesive energy is gained -57.61 meV/atom.) which is similar to our ABi model. Because of the high stability of ABi model, we continue our investigations only with $\mathrm{ABi}$ model. From now on, when we refer to $\mathrm{Sb} / \mathrm{Bi}$ $\mathrm{HS}$, we mean the ABi model of the $\mathrm{Sb} / \mathrm{Bi}$ heterostructure.

\subsection{The Electronic properties of the $\mathrm{Sb} / \mathrm{Bi} \mathrm{HS}$}

\subsubsection{The pristine structure}

The calculated band structure for $\mathrm{Sb} / \mathrm{Bi} \mathrm{HS}$ (Fig.7) shows that this material is a semiconductor with an indirect bandgap of $159 \mathrm{meV}$ at GGA and a semimetal at GGA+SOC (SOGGA) level of theory. According to the heavy atoms consisting the HS, especially Bismuth, the determinant influence of SOC interactions are expectable. Generally, considering the SOC effect is important in structures with heavy elements [37], but little and neglectable for lighter ones [38]. As we know, considering the SOC eliminates the energy degeneration and assets the electronic properties more precisely. Therefore, it is expected that in case of potential experimental research, the results would be more close to our SOGGA solutions. Therefore, in all of our electronic and optical calculations, we continued at the SOGGA level of theory.

It can be seen from the atom projected density of states (Fig.7b) that in the valance band the contribution of $\mathrm{Bi}$ atoms is slightly higher than $\mathrm{Sb}$ ones but in the conduction band, they play the same role in the electronic properties. Besides, the orbital projected density of states (Fig.7c) represents that the 

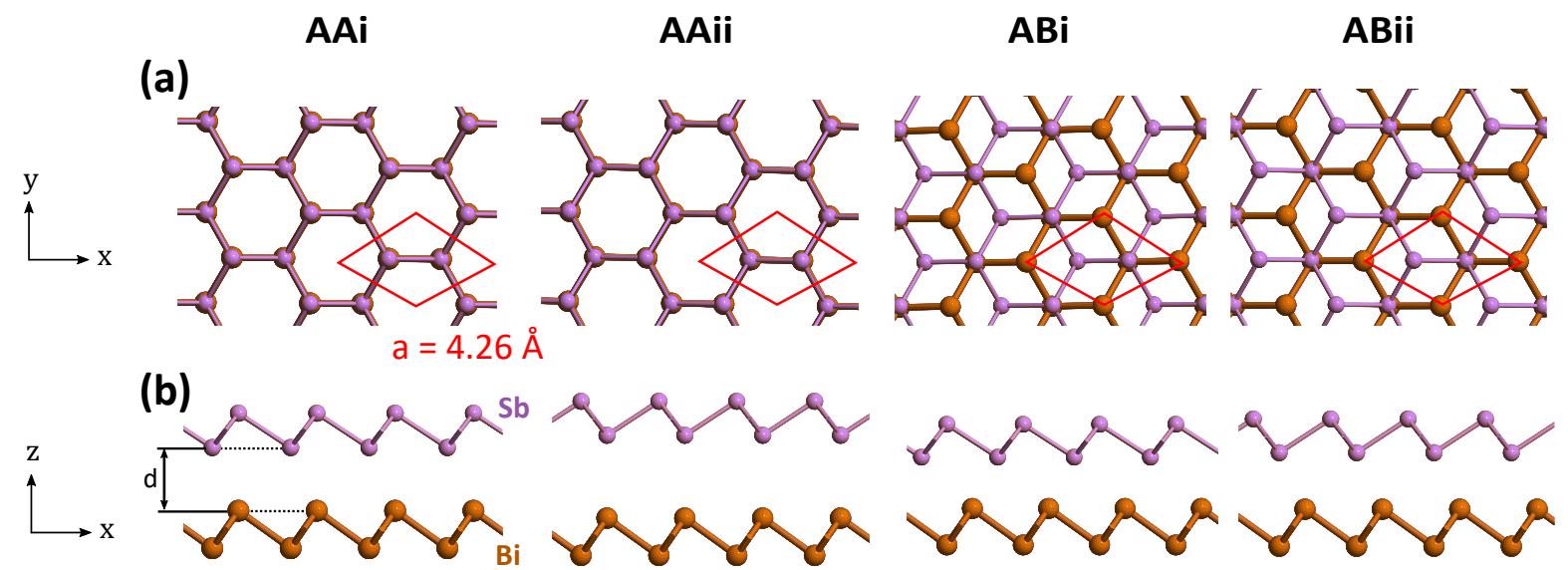

(c)
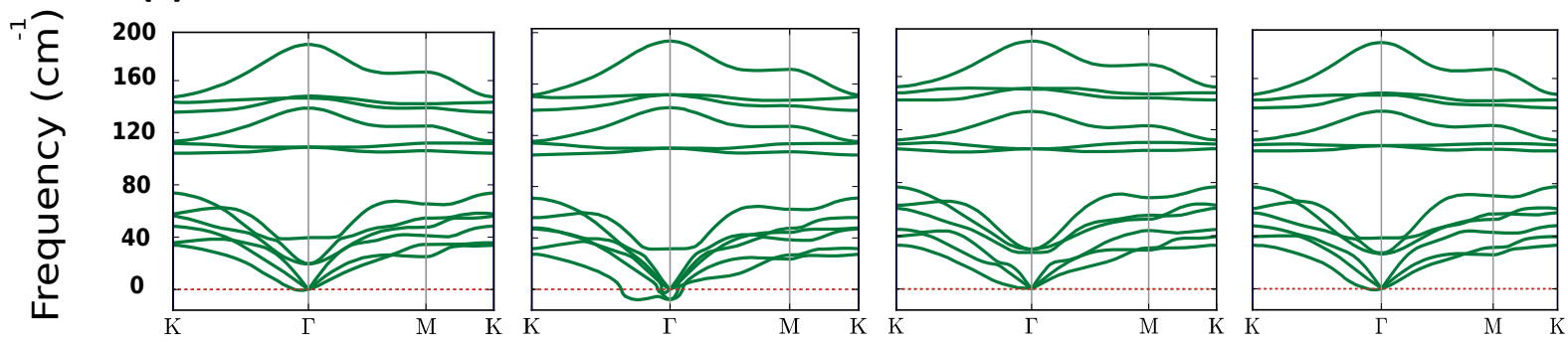

Figure 5: Four models of Sb/Bi HS. (a) The top view, (b) the side view, and (c) the phonon dispersion for each model.

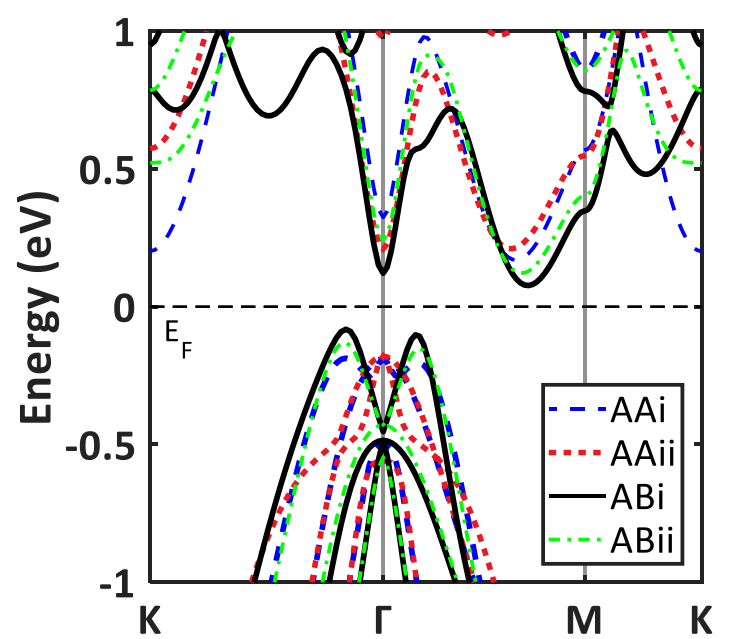

Figure 6: GGA band structures of the four models of $\mathrm{Sb} / \mathrm{Bi} \mathrm{HS}$.

contribution of $\mathrm{p}$ orbitals is determinant and other orbitals have neglectable roles. Considering that both consisting elements are in group $\mathrm{V}$, the influence of $\mathrm{p}$ orbitals is natural. Within the whole showed range in the conduction band, and near the Fermi level in the valance band, the $\mathrm{Sb}$ and $\mathrm{Bi}$ p orbitals have a superposition, therefore the mechanism in which the layers connect to each other may be through orbital hybridization. There are similar reports about the superposition of orbital projected density of states in Van-der Waals heterostructures, and exis- tence of orbital hybridization between the layers; such as Antimonene/Arsenene and Antimonene/Germanene [26, 28].

To have further insight into the electronic properties of the HS, we calculated the electron density and electron localization function (ELF) [39, 40] (Fig.8). Electron density can bring information about how electrons are distributed in the lattice, and ELF allows evaluating the chemical interactions from the charge localization between individual atoms. Our calculations show that there is a little and uniform electron density of about $0.3 \AA^{-3}$ (Fig.8a) between atoms and layers. Electron density is significant around atoms and it decreases with going far from them. The maximum electron density is collected around $\mathrm{Sb}$ atoms with a value of $10 \AA^{-3}$ and subsequently $\mathrm{Bi}$ atoms have an electron density of $5 \AA^{-3}$ around them (Fig.8b). Considering that electron density around $\mathrm{Sb}$ atoms is one time larger than around $\mathrm{Bi}$ atoms, it could be said that some spatial separation of electrons and holes may occur in the HS. In other words, most of the electrons exist in the Sb layer and most of the holes dominate the Bi layer. Such separation would decrease the recombinations of photo-generated electrons and holes in solar cells and dramatically increase their efficiency. That phenomenon has also been observed in heterostructures like InSe/Sb, $\mathrm{MoS}_{2} / \mathrm{MoSe}_{2}$, and $\mathrm{MoS}_{2} / \mathrm{ReS}_{2}$ [41-43].

It can be seen from the ELF (Fig.8c) that the highest localization is above the upper $\mathrm{Sb}$ atoms and subsequently, under the lower $\mathrm{Bi}$ atoms. There is no localization in the shortest distance between the upper and the lower layers. Therefore, no chemical covalent bonds exist between the layers and other mechanisms like Van-der Waals's interactions or orbitals hybridization may 


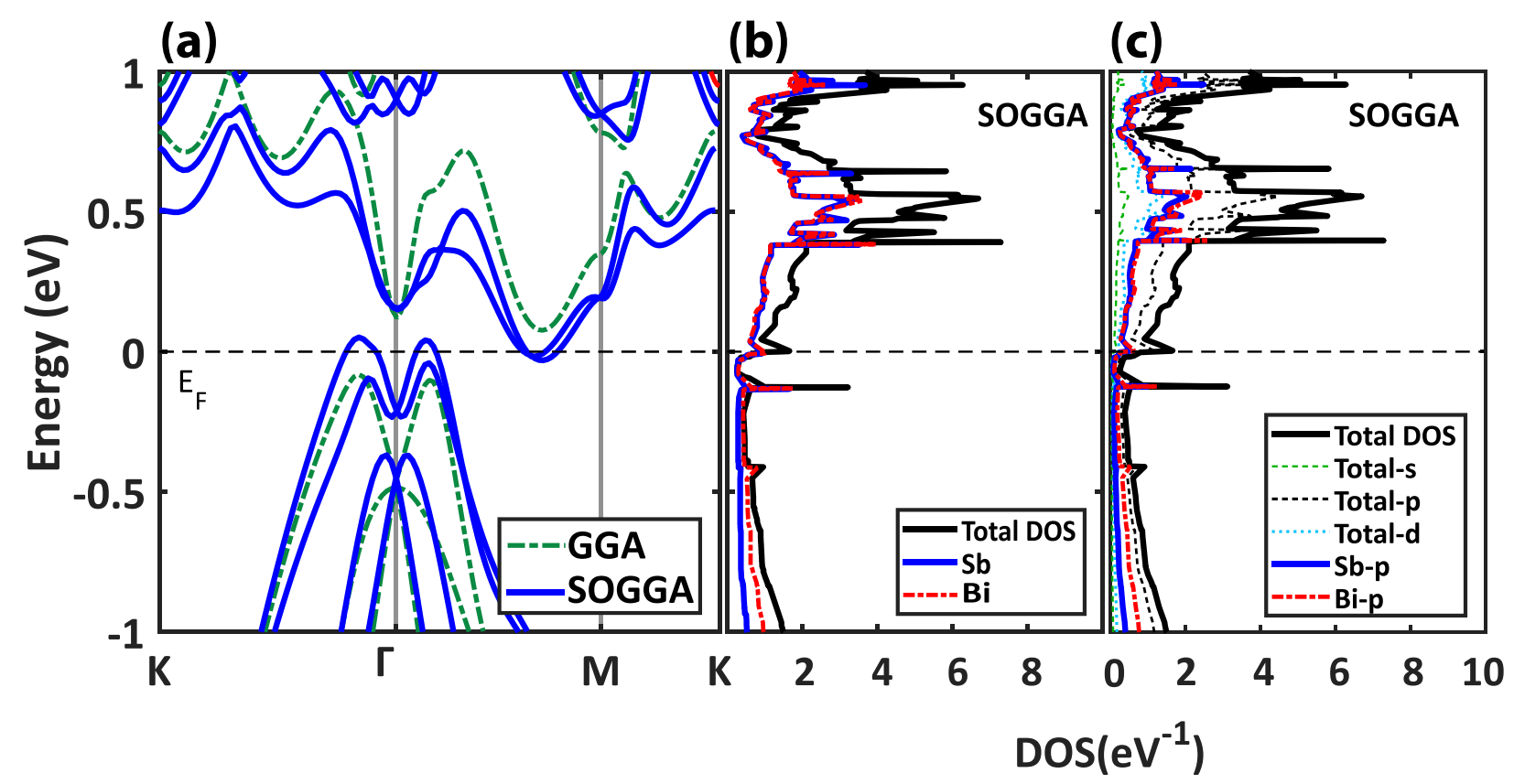

Figure 7: The Electronic properties of the Sb/Bi HS. (a) Band structure at GGA and SOGGA levels of theory. (b,c) Atom and orbital projected density of states, respectively, both at SOGGA level of theory.

connect the layers. The lack of localization and the absence of covalent bonds between the layers are also reported in the previous heterostructures like $\mathrm{G} / \mathrm{Sb}, \mathrm{PdTe}_{2} / \mathrm{Sb}[28,44]$ as well as Antimonene grown on (111) Ag substrate [45].

\subsubsection{Appying Electric Field and Strain}

As mentioned before, our calculations show that the $\mathrm{Sb} / \mathrm{Bi}$ HS is a semimetal at SOGGA level of theory. Existence of a bandgap in a 2D material would bring hope for designing nanoscale field-effect transistors (FETs). Of the methods for opening a bandgap in non-gap materials are applying an external electric field and/or tensile and compressive strain. For example, Li et al have tunned the bandgap of $\mathrm{MoS}_{2} / \mathrm{Sb}$ heterostructure from 0 to $1.2 \mathrm{eV}$ by applying external electric field [46]. Lu et al have also reported that with applying tensile strain, the bandgap of h-BN/Sb heterostructure increases [26]. Besides, Dong at al. could tune the band gap of $\mathrm{As} / \mathrm{MX}_{2}(\mathrm{X}=\mathrm{S}, \mathrm{Se} \& \mathrm{M}=\mathrm{Mo}, \mathrm{W})$ heterostructures from 0 to $1.5 \mathrm{eV}$ by applying an electric field from -8 to $4 \mathrm{~V} / \mathrm{nm}$ (with positive direction from As to $\mathrm{MX}_{2}$ ) and biaxial strain from -10 to $15 \%$ [47]. We also applied electric field and biaxial strain to tune the bandgap of $\mathrm{Sb} / \mathrm{Bi} \mathrm{HS}$. In applying the electric field, we chose the positive direction from $\mathrm{Bi}$ to $\mathrm{Sb}$ layer and vise versa and applied the electric field from -8 to $8 \mathrm{~V} / \mathrm{nm}$ (Fig.9b). We also applied the biaxial strain within the range from -14 to $+14 \%$ with steps of $2 \%$.

By use of electric field, the GGA bandgap of the HS can be tuned within the range from $146 \mathrm{meV}$ to $168 \mathrm{meV}$. It increases with the negative electric fields and decreases with the positive ones (Fig.9). Also by applying tensile strains, the bandgap encloses immediately and by applying compressive strain, the bandgap reaches to the value of around $76 \mathrm{meV}$ under the strain of $-2 \%$ and finally encloses under the strain of $-4 \%$. Our inves- tigations at SOGGA level of theory shows that the electronic properties of the material are robust against the electric field and strain and can not be tuned trough these methods. Considering the importance of the SOC interactions in this HS, it could be summarised that it is a semimetal in which the CBM and VBM touch the Fermi level. Most of the electrons are collected in the Sb layer and most of the holes rule the Bi layer. The semimetallic properties are robust against applying the electric field and strain and would not change with such external factors.

\subsection{Mechanical Properties of the Sb/Bi HS}

With applying strain, a material resists and stress applies to its surface. As the applied strain increases, the stress gets greater until under a certain critical strain the material cracks and the stress suddenly drops. The more the so-called critical strain would be, the more the material can be stretched or compressed. In addition, the cohesive energy between layers tells about how much the layers tend to be integrated and connected to each other. By considering the stress-strain and cohesive energy-strain relationships, one can find out how much a material can be stretched and/or compressed, and remain stable. Fig.10a shows the stress-strain and cohesive energy-strain curves for the $\mathrm{Sb} / \mathrm{Bi} \mathrm{HS}$. The stress is $0.28 \mathrm{~N} / \mathrm{m}$ in the pristine structure and by the increase of tensile strain, it begins to increase slightly. Under the strain of $16 \%$, it reaches to $5.08 \mathrm{~N} / \mathrm{m}$ and then drops. Therefore, the critical strain and the respective ideal strength are $16 \%$ and $5.08 \mathrm{~N} / \mathrm{m}$, respectively. Besides, the cohesive energy is $-173 \mathrm{meV} /$ atom in the pristine structure, which by the increase of tensile strain exponentially increases and finally reaches to its maximum negative value of $-21 \mathrm{meV} /$ atom and then gets positive. The positiveness of the cohesive energy means that the mechanism of formation of the 

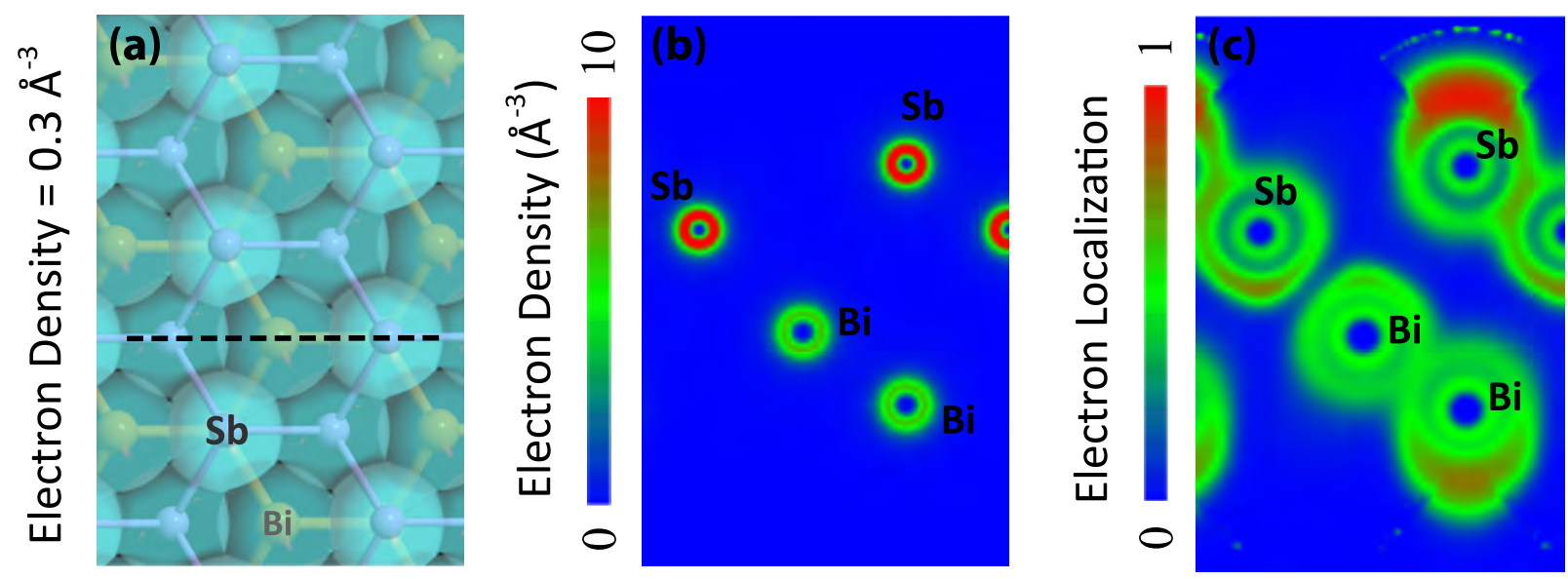

Figure 8: (a) Top view of the overall electron density with an iso-value of $0.6 \AA^{-3}$. (b,c) Electron density and ELF of the cross section along the dashed line in (a), respectively. All three were calculated at SOGGA level of theory.

(a)

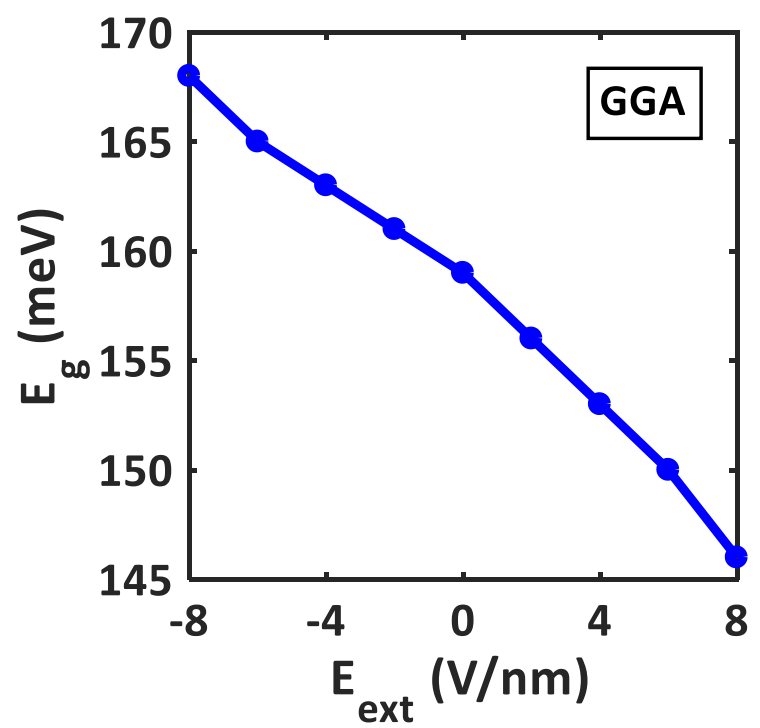

(b)

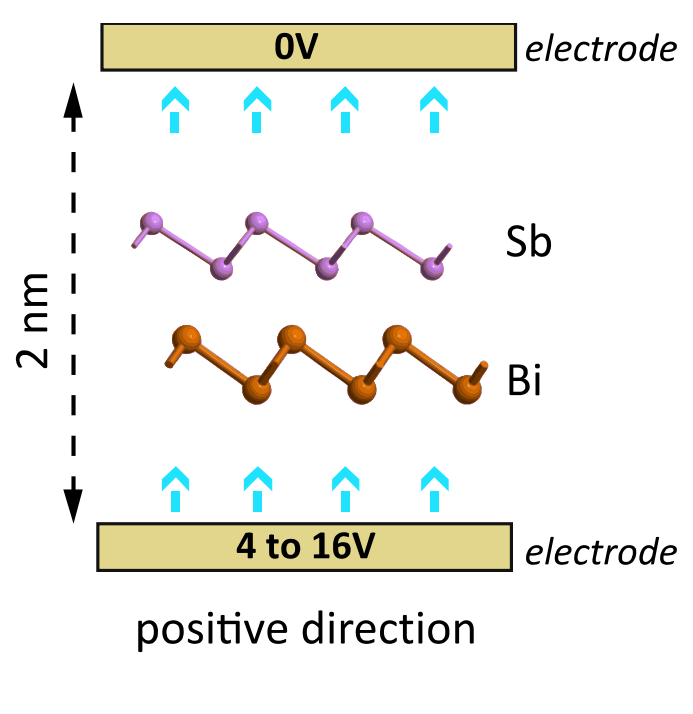

Figure 9: (a) Variation of the Sb/Bi HS GGA bandgap, by applying external electric field. (b) Schematic of applying external filed in the positive direction.

HS becomes endothermic and the connection between the layers would be canceled. Moreover, the phonon dispersion calculations show that the material is still stable under the strain of $14 \%$ (Fig.11). Intuitively, we predict that under the tensile strain of $10 \%$ the $\mathrm{Sb}$ and $\mathrm{Bi}$ layers separate and under the strain of $16 \%$, the material cracks. With applying compressive strain, the stress descends towards the negative direction and it reaches to $-3.67 \mathrm{~N} / \mathrm{m}$ under the strain of $-8 \%$, and after that it suddenly ascends. Therefore, we took the $-8 \%$ as the critical compressive strain with a corresponding compressive ideal strength of -3.67 $\mathrm{N} / \mathrm{m}$. It should be noted that the cohesive energy is negative within this range, that is, the layers are connected to each other.

For further investigations about the mechanical properties, we calculated Young's modulus, which is defined by the equa- tions below,

$$
E=\frac{\sigma}{\epsilon}
$$

where, $\epsilon$ and $\sigma$ are the strain and stress, respectively. According to the equation (4), the more Young's modulus is, the greater the response of the material to the applied strain would be. That is, the material resists more against and has less intention for being stretched or compressed. To calculate Young's modulus, we applied strains within the range of $-2 \%$ to $2 \%$ with steps of $0.5 \%$. The variations of the stress in this range of strain is approximately linear. Furthermore, we fitted the computational data with a linear function (Fig.10b). By calculating the gradiant of the linear fitted function, we anticipate Young's modulus of $64.3 \% \mathrm{~N} / \mathrm{m}$ for the HS. 
(a)

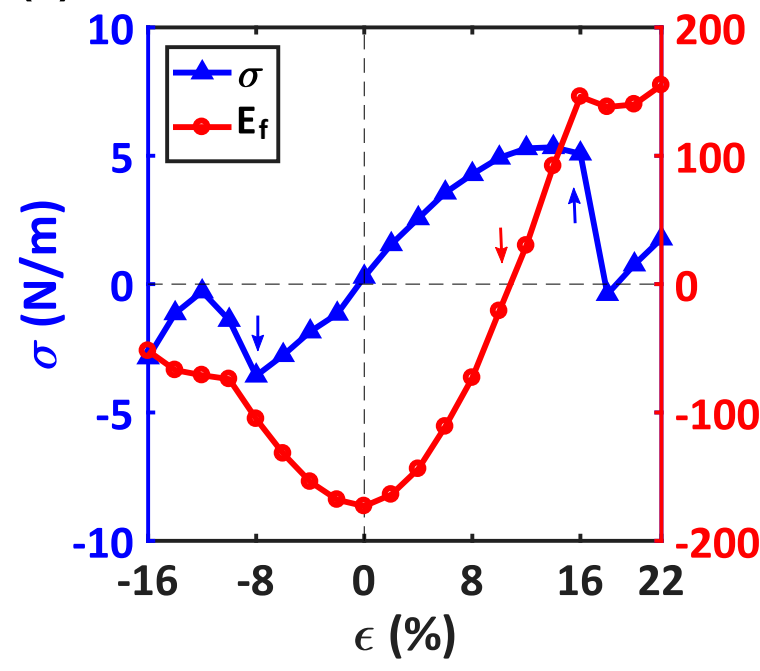

(b)

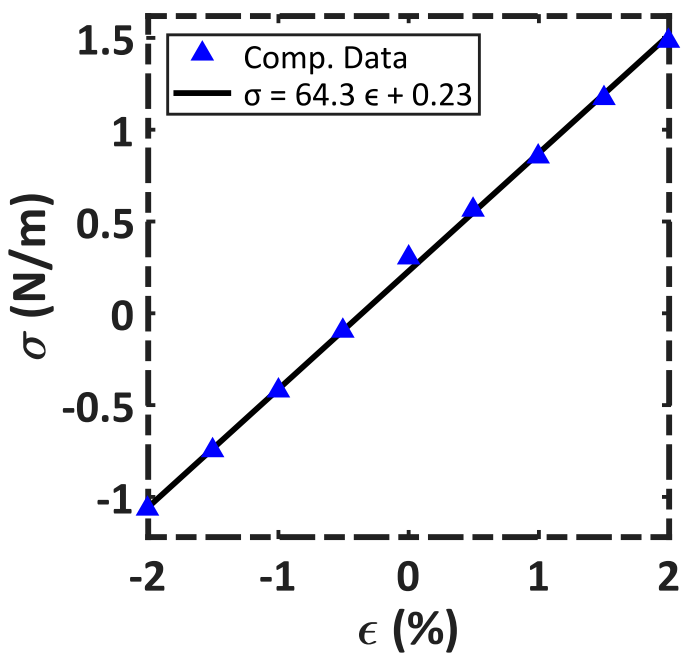

Figure 10: The Mechanical properties of Sb/Bi HS. (a) Curves of stress and cohesive energies variations by strain. (b) The stress-strain curve within the strain range of $-2 \%$ to $2 \%$, with a fitted function used for calculating Young's modulus.

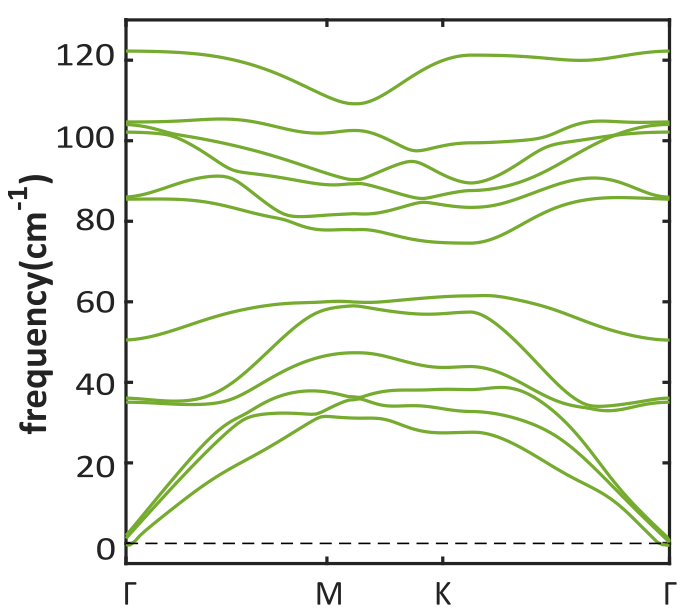

Figure 11: Phonon dispersion spectrum for the HS under the strain of $14 \%$. No imaginary modes are visible, therefore the structure is stable.

We compared our mechanical results about the HS with other 2D materials including Arsenene, Antimonene, Silicene, Graphene, and Molibdanium disulfide $\left(\mathrm{MoS}_{2}\right)$ (Table 3). In comparison with group V 2D materials, the critical strain of the HS is bigger than Phosphorene's but smaller than Antimonene's and Arsenene's. Interestingly, Young's modulus of the HS is greater than all of the group V 2D materials' and even than Silicene's. Therefore, although the HS loses its stability in strains longer than $10 \%$, its high Young's modulus do not allow for size change so easily.

\subsection{Optical Properties of the $\mathrm{Sb} / \mathrm{Bi} H \mathrm{HS}$}

The optical properties are described by the complex dielectric function, $\epsilon(\omega)=\epsilon_{1}(\omega)+i \epsilon_{2}(\omega)$. We first calculated the
Table 3: The mechanical properties of the $\mathrm{Sb} / \mathrm{Bi} \mathrm{HS}$ including critical strain $\left(\epsilon^{*}\right)$, ideal strength $\left(\sigma^{*}\right)$, and Young's modulus $\left(E_{x y}\right)$ compared with other 2D materials.

\begin{tabular}{llll}
\hline 2D material & $\epsilon^{*}(\%)$ & $\sigma^{*}(N / m)$ & $E_{x y}(N / m)$ \\
\hline Phosphorene & $8[48]$ & $20.26[48]$ & $23[49]$ \\
Antimonene [35] & 20 & 3.75 & 32.9 \\
Arsenene [35] & 17 & 5.15 & 52.8 \\
Bismuthene[50] & 15 & n/a & 26.25 \\
Silicene [51] & 17.5 & 7.20 & 61.7 \\
Sb/Bi HS (this study) & 16 & 5.08 & 64.3 \\
MoS 2 & $28[52]$ & $15.73[52]$ & $118[53]$ \\
Graphene & $19[54]$ & $32.93[54]$ & $345[55]$ \\
\hline \hline
\end{tabular}

susceptibility tensor by Kubo-Greenwood formula [56],

$$
\chi_{i j}(\omega)=-\frac{e^{2} \hbar^{4}}{m^{2} \epsilon_{0} V \omega^{2}} \sum \frac{f\left(E_{m}\right)-f\left(E_{n}\right)}{E_{n m}-\hbar \omega-i \Gamma} \pi_{n m}^{i} \pi_{m n}^{j}
$$

where $\pi_{n m}^{i}$ is the $\mathrm{i}$-th component of the dipole matrix between state $\mathrm{n}$ and $\mathrm{m}, \mathrm{V}$ the volume, $\Gamma$ the broadening, and $\mathrm{f}$ the Fermi function. Subsequently, one may calculate the dielectric function by the equation below,

$$
\epsilon(\omega)=1+\chi(\omega)
$$

Furthermore, the optical absorption coefficient $(\alpha)$ can be calculated through,

$$
\alpha=2 \frac{\omega}{c} \sqrt{\frac{\sqrt{\epsilon_{1}^{2}+\epsilon_{2}^{2}}-\epsilon_{1}}{2}}
$$

where $\mathrm{c}$ is the speed of light.

Fig. 12 shows the real and imaginary parts of the dielectric function as well as the optical absorption of the $\mathrm{Sb} / \mathrm{Bi} \mathrm{HS}$. In 

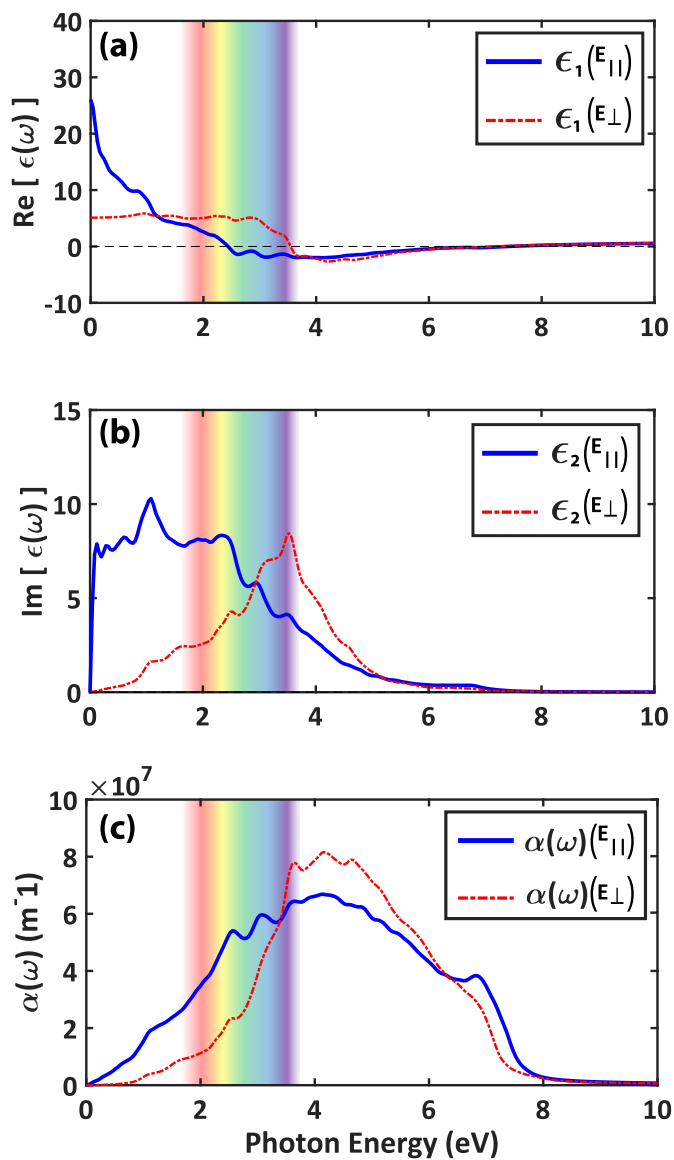

Figure 12: Optical properties of Sb/Bi HS. (a) Real and (b) imaginary parts of the dielectric function. (c) Optical absorption coefficient.

these calculations, we used the GGA with considerations of the SOC interactions (SOGGA). For the isotropy of the crystalline structure of the HS in the xy-plane, the dielectric functions for linear polarization along $\mathrm{x}$ and $\mathrm{y}$ direction are similar $\left(\epsilon^{x x}=\epsilon^{y y}\right)$. Therefore we note the optical properties for the parallel ( $E_{\|}$, along with the $\mathrm{x}$ - and y-direction) and perpendicular $\left(E_{\perp}\right.$, along with the $\mathrm{z}$-direction) polarization regarding the sheet plane.

As can be seen in Fig.12, the behavior of the HS is different for different polarization. To the best of our knowledge, the negative values of the real part of the dielectric function show a metallic optical properties. As can be seen from Fig. 12a there are negative values for the real part of the dielectric function in the UV spectrum which stands for metallic properties. The beginning of the metallic properties differs in different polarization. The HS begins to be metallic from $3.56 \mathrm{eV}$ (348 $\mathrm{nm})$ in the UV and $2.44 \mathrm{eV}(548 \mathrm{~nm})$ in the visible region for the perpendicular and parallel polarization, respectively. The metallic characteristics are also reported for monolayer Antimonene [23, 24] but unlike the $\mathrm{Sb} / \mathrm{Bi} \mathrm{HS}$, it begins in the UV region, not visible.

Peaks in the imaginary part of the dielectric function are due to absorption of the incident photons and direct transitions of

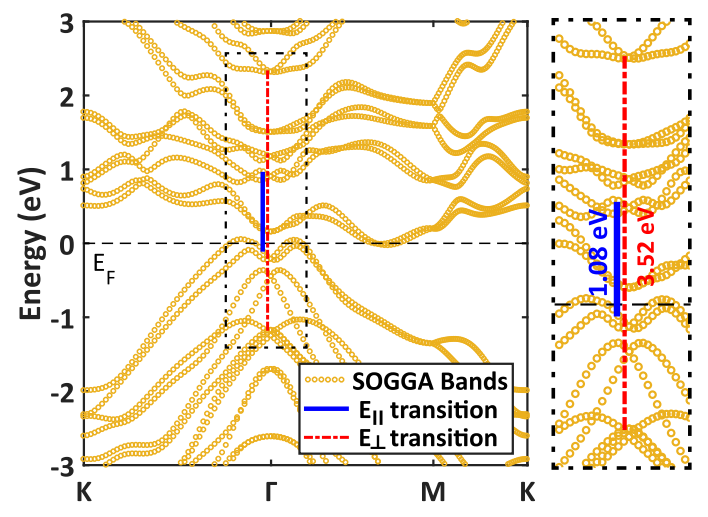

Figure 13: Direct electron transitions between bands below and above the Fermi level, for parallel $(1.08 \mathrm{eV})$ and perpendicular $(3.52 \mathrm{eV})$ polarization.

the electrons between bands below and above the Fermi energy [57]. As can be seen from Fig.12b there are two major peaks in the imaginary part of the dielectric function in 1.08 and 3.52 $\mathrm{eV}$, respectively. By analyzing the band structure and precise measuring of the distance between bands below and above the Fermi level in different $\mathrm{k}$ points, one can make a relative assessment about how the electron transitions take place. Our analysis shows that these transitions for perpendicular and parallel polarization take place at the $\Gamma$ point, where is the only point which the energy difference between some conduction and valance bands are exactly equal to transition energies (Fig.13). Direct electron transitions are also reported for the monolayer Antimonene in $3.4,4.7,5.9,6.9$, and $8.0 \mathrm{eV}$ at different $\mathrm{k}$ points [23].

Fig.12c shows the absorption coefficient for the $\mathrm{Sb} / \mathrm{Bi} \mathrm{HS}$. In the infrared region, the absorption is not so much, but it is a bit more than the monolayer Antimonene [23]. Therefore, compared with the monolayer Antimonene, the HS absorbs more heat. The parallel and perpendicular polarization are absorbed deeper in the visible and UV regions, respectively. The maximum of the absorption is about $8 \times 10^{7}$ for photon energy of $\sim 4 \mathrm{eV}(310 \mathrm{~nm})$ in the UV region. Our investigations show that the absorption in the visible region for the HS is sort of 4 times greater than the monolayer Antimonene, in which it is reported neglectable [24]. Such increase in the absorption in the visible region is also reported in $\mathrm{Sb} / \mathrm{GaAs}$ heterostructure [58].

In general, the dependence of reflectance and absorption on the polarization of the incident light, along with the increase of absorption of perpendicular polarization in the visible region can improve the applications of the $\mathrm{Sb} / \mathrm{Bi} \mathrm{HS}$ in beam splitters and nano-scale mirrors compared with the monolayer Antimonene.

\section{Conclusion}

In summary, we systematically calculated the structural, electronic, mechanical, and optical properties of the $\mathrm{Sb} / \mathrm{Bi}$ heterostructure using the first principle density functional theory. Our investigations show that this heterostructure is theoretically stable 
and possible, and the $\mathrm{ABi}$ model is the most stable stacking. Its semimetallic electronic characteristics are robust against external factors such as electric field and mechanical strain. The $\mathrm{Sb} / \mathrm{Bi}$ heterostructure is mechanically a stiff material with Young's modulus of $64.3 \mathrm{~N} / \mathrm{m}$ which is larger than all known group $\mathrm{V}$ monolayers' and even than Silicene's. The optical properties of the HS are dependant on the polarization of the incident light. It begins to be metallic in the visible and UV region for parallel and perpendicular polarization, respectively. In comparison with monolayer Antimonene, the absorption of the perpendicular polarization in the visible region is improved. The heterostructure has a good optical absorption and reflectance in the visible and UV region. In addition, the polarization of the incident light makes significant difference in the optical propertie. Therefore we predict its potential applications in beam splitters and nano-scale mirrors.

\section{Acknowledgment}

We acknowledge Mohammad Ali Mohebpour and Salimeh Mahdavifar for helpful discussions and recommendations. We are thankful to the Research Council of the University of Guilan for the partial support of this research.

\section{Declaration of Interests}

The authors declare that they have no known competing financial interests or personal relationships that could have appeared to influence the work reported in this paper.

\section{References}

[1] W. Lu, H. Nan, J. Hong, Y. Chen, C. Zhu, Z. Liang, X. Ma, Z. Ni, C. Jin, Z. Zhang, Plasma-assisted fabrication of monolayer phosphorene and its raman characterization, Nano Res. 7 (6) (2014) 853-859.

[2] I. W. Frank, D. M. Tanenbaum, A. M. van der Zande, P. L. McEuen, Mechanical properties of suspended graphene sheets, J. Vac. Sci. Technol. B 25 (6) (2007) 2558-2561.

[3] K. S. Novoselov, V. Fal, L. Colombo, P. Gellert, M. Schwab, K. Kim, et al., A roadmap for graphene, nature 490 (7419) (2012) 192.

[4] K. S. Novoselov, A. K. Geim, S. V. Morozov, D. Jiang, Y. Zhang, S. V. Dubonos, I. V. Grigorieva, A. A. Firsov, Electric field effect in atomically thin carbon films, Science 306 (5696) (2004) 666-669.

[5] A. K. Geim, Random walk to graphene (nobel lecture), Angew. Chem. Int. Ed. 50 (31) (2011) 6966-6985.

[6] C. Kamal, M. Ezawa, Arsenene: Two-dimensional buckled and puckered honeycomb arsenic systems, Phys. Rev. B 91 (8) (2015) 085423.

[7] S. Zhang, Z. Yan, Y. Li, Z. Chen, H. Zeng, Atomically thin arsenene and antimonene: semimetalsemiconductor and indirectdirect bandgap transitions, Angew. Chem. Int. Ed. 54 (10) (2015) 3112-3115.

[8] Z. Ni, Q. Liu, K. Tang, J. Zheng, J. Zhou, R. Qin, Z. Gao, D. Yu, J. Lu, Tunable bandgap in silicene and germanene, Nano Lett. 12 (1) (2011) 113-118.

[9] M. E. Dvila, L. Xian, S. Cahangirov, A. Rubio, G. Le Lay, Germanene: a novel two-dimensional germanium allotrope akin to graphene and silicene, New J. Phys. 16 (9) (2014) 095002.

[10] M. Chhowalla, H. S. Shin, G. Eda, L.-J. Li, K. P. Loh, H. Zhang, The chemistry of two-dimensional layered transition metal dichalcogenide nanosheets, Nat. Chem. 5 (4) (2013) 263.

[11] L. Liu, Y. P. Feng, Z. X. Shen, Structural and electronic properties of h-bn, Phys. Rev. B 68 (10) (2003) 104102.
[12] A. Bafekry, M. Ghergherehchi, S. F. Shayesteh, Tuning the electronic and magnetic properties of antimonene nanosheets via point defects and external fields: first-principles calculations, Phys. Chem. Chem. Phys. 21 (20) (2019) 10552-10566.

[13] M. A. Mohebpour, M. Saffari, H. R. Soleimani, M. B. Tagani, High performance of mixed halide perovskite solar cells: Role of halogen atom and plasmonic nanoparticles on the ideal current density of cell, Physica E 97 (2018) 282-289.

[14] Y. Xu, C.-Y. Hsieh, L. Wu, L. Ang, Two-dimensional transition metal dichalcogenides mediated long range surface plasmon resonance biosensors, J. Phys. D 52 (6) (2018) 065101.

[15] D. Reddy, L. F. Register, G. D. Carpenter, S. K. Banerjee, Graphene fieldeffect transistors, J. Phys. D 44 (31) (2011) 313001.

[16] A. A. Erchak, D. J. Ripin, S. Fan, P. Rakich, J. D. Joannopoulos, E. P. Ippen, G. S. Petrich, L. A. Kolodziejski, Enhanced coupling to vertical radiation using a two-dimensional photonic crystal in a semiconductor light-emitting diode, Appl. Phys. Lett. 78 (5) (2001) 563-565.

[17] M. Saffari, M. A. Mohebpour, H. R. Soleimani, M. B. Tagani, Dft analysis and fdtd simulation of ch3nh3pbi3- $\mathrm{x} \mathrm{cl} \mathrm{x} \mathrm{mixed} \mathrm{halide} \mathrm{perovskite}$ solar cells: role of halide mixing and light trapping technique, J. Phys. D 50 (41) (2017) 415501.

[18] Y. Cai, S. Liu, X. Yin, Q. Hao, M. Zhang, T. Wang, Facile preparation of porous one-dimensional mn2o3 nanostructures and their application as anode materials for lithium-ion batteries, Physica E 43 (1) (2010) 70-75.

[19] G. Wang, R. Pandey, S. P. Karna, Atomically thin group v elemental films: theoretical investigations of antimonene allotropes, ACS Appl. Mater. Interfaces 7 (21) (2015) 11490-6. doi : 10.1021/acsami . 5b02441.

[20] O. z. Aktrk, V. O. zelik, S. Ciraci, Single-layer crystalline phases of antimony: Antimonenes, Phys. Rev. B 91 (23) (2015) 235446.

[21] E. Aktrk, O. z. Aktrk, S. Ciraci, Single and bilayer bismuthene: Stability at high temperature and mechanical and electronic properties, Phys. Rev. B 94 (1) (2016) 014115.

[22] C. Gibaja, D. RodriguezSanMiguel, P. Ares, J. GmezHerrero, M. Varela, R. Gillen, J. Maultzsch, F. Hauke, A. Hirsch, G. Abelln, Fewlayer antimonene by liquidphase exfoliation, Angew. Chem. Int. Ed. 55 (46) (2016) 14345-14349.

[23] D. Singh, S. K. Gupta, Y. Sonvane, I. Lukaevi, Antimonene: a monolayer material for ultraviolet optical nanodevices, J. Mater. Chem. C 4 (26) (2016) 6386-6390.

[24] Y. Xu, B. Peng, H. Zhang, H. Shao, R. Zhang, H. Zhu, Firstprinciple calculations of optical properties of monolayer arsenene and antimonene allotropes, Ann. Phys. 529 (4) (2017) 1600152

[25] S.-D. Guo, J. Dong, Biaxial tensile strain tuned up-and-down behavior on lattice thermal conductivity in $\beta$-asp monolayer, J. Phys. D 51 (26) (2018) 265307.

[26] H. Lu, J. Gao, Z. Hu, X. Shao, Biaxial strain effect on electronic structure tuning in antimonene-based van der waals heterostructures, RSC Adv. 6 (104) (2016) 102724-102732. doi:10.1039/c6ra21781h.

[27] M.-L. Tsai, S.-H. Su, J.-K. Chang, D.-S. Tsai, C.-H. Chen, C.-I. Wu, L.-J. Li, L.-J. Chen, J.-H. He, Monolayer mos 2 heterojunction solar cells, ACS Nano 8 (8) (2014) 8317-8322.

[28] X. Chen, Q. Yang, R. Meng, J. Jiang, Q. Liang, C. Tan, X. Sun, The electronic and optical properties of novel germanene and antimonene heterostructures, J. Mater. Chem. C 4 (23) (2016) 5434-5441. doi: 10. 1039/c6tc01141a.

[29] J. M. Soler, E. Artacho, J. D. Gale, A. García, J. Junquera, P. Ordejón, D. Sánchez-Portal, The siesta method for ab initio order-n materials simulation, J. Phys. Condens. Matter 14 (11) (2002) 2745.

[30] J. P. Perdew, K. Burke, M. Ernzerhof, Generalized gradient approximation made simple, Phys. Rev. Lett. 78 (7) (1997) 1396. doi:10.1103/ PhysRevLett.78.1396.

[31] S. Grimme, Accurate description of van der waals complexes by density functional theory including empirical corrections, J. Comput. Chem. 25 (12) (2004) 1463-1473.

[32] N. Mounet, M. Gibertini, P. Schwaller, D. Campi, A. Merkys, A. Marrazzo, T. Sohier, I. E. Castelli, A. Cepellotti, G. Pizzi, N. Marzari, Twodimensional materials from high-throughput computational exfoliation of experimentally known compounds, Nat. Nanotechnol. 13 (3) (2018) 246252. doi: 10.1038/s41565-017-0035-5.

[33] S. Zhang, M. Xie, F. Li, Z. Yan, Y. Li, E. Kan, W. Liu, Z. Chen, H. Zeng, Semiconducting group 15 monolayers: a broad range of band gaps and 
high carrier mobilities, Angew. Chem. Int. Ed. 55 (5) (2016) 1666-1669.

[34] M.-Y. Liu, Y. Huang, Q.-Y. Chen, Z.-Y. Li, C. Cao, Y. He, Strain and electric field tunable electronic structure of buckled bismuthene, RSC Adv. 7 (63) (2017) 39546-39555.

[35] G. Liu, Z. Gao, J. Zhou, Strain effects on the mechanical properties of group-v monolayers with buckled honeycomb structures, Physica E 112 (2019) 59-65.

[36] M. Zhao, X. Zhang, L. Li, Strain-driven band inversion and topological aspects in antimonene, Sci. Rep. 5 (2015) 16108.

[37] S. Manzeli, D. Ovchinnikov, D. Pasquier, O. V. Yazyev, A. Kis, 2d transition metal dichalcogenides, Nat. Rev. Mater.s 2 (8) (2017) 17033.

[38] M. Ezawa, Triplet fermions and dirac fermions in borophene, Phys. Rev. B 96 (3) (2017) 035425.

[39] A. D. Becke, K. E. Edgecombe, A simple measure of electron localization in atomic and molecular systems, J. Chem. Phys. 92 (9) (1990) 53975403.

[40] A. Savin, O. Jepsen, J. Flad, O. K. Andersen, H. Preuss, H. G. von Schnering, Electron localization in solidstate structures of the elements: the diamond structure, Angew. Chem. Int. Ed. 31 (2) (1992) 187-188.

[41] Z. Zhang, Y. Zhang, Z. Xie, X. Wei, T. Guo, J. Fan, L. Ni, Y. Tian, J. Liu, L. Duan, Tunable electronic properties of an sb/inse van der waals heterostructure by electric field effects, PCCP 21 (10) (2019) 5627-5633.

[42] M. Z. Bellus, M. Li, S. D. Lane, F. Ceballos, Q. Cui, X. C. Zeng, H. Zhao, Type-i van der waals heterostructure formed by mos 2 and res 2 monolayers, Nanoscale Horiz. 2 (1) (2017) 31-36.

[43] F. Ceballos, M. Z. Bellus, H.-Y. Chiu, H. Zhao, Ultrafast charge separation and indirect exciton formation in a mos 2 mose 2 van der waals heterostructure, ACS Nano 8 (12) (2014) 12717-12724.

[44] X. Wu, Y. Shao, H. Liu, Z. Feng, Y. Wang, J. Sun, C. Liu, J. Wang, Z. Liu, S. Zhu, Epitaxial growth and airstability of monolayer antimonene on pdte2, Adv. Mater. 29 (11) (2017) 1605407.

[45] Y. Shao, Z.-L. Liu, C. Cheng, X. Wu, H. Liu, C. Liu, J.-O. Wang, S.-Y. Zhu, Y.-Q. Wang, D.-X. Shi, Epitaxial growth of flat antimonene monolayer: A new honeycomb analogue of graphene, Nano Lett. 18 (3) (2018) 2133-2139.

[46] W. Li, Y. Ma, X. Wang, X. Dai, Electric field effects on the electronic structures of mos2/antimonene van der waals heterostructure, Solid State Commun. 293 (2019) 28-32.

[47] M. M. Dong, C. He, W. X. Zhang, Tunable electronic properties of arsenene and transition-metal dichalcogenide heterostructures: a firstprinciples calculation, J. Phys. Chem. C 121 (40) (2017) 22040-22048.

[48] Q. Wei, X. Peng, Superior mechanical flexibility of phosphorene and fewlayer black phosphorus, Appl. Phys. Lett. 104 (25) (2014) 251915.

[49] Y. Zhao, A. M. Nardes, K. Zhu, Solid-state mesostructured perovskite ch3nh3pbi3 solar cells: charge transport, recombination, and diffusion length, J. Phys. Chem. Lett. 5 (3) (2014) 490-494.

[50] P. Aghdasi, R. Ansari, S. Rouhi, M. Goli, On the elastic and plastic properties of the bismuthene adsorbed by h, f, cl and br atoms, Superlattice. Microst. (2019) 106242.

[51] B. Mortazavi, O. Rahaman, M. Makaremi, A. Dianat, G. Cuniberti, T. Rabczuk, First-principles investigation of mechanical properties of silicene, germanene and stanene, Physica E 87 (2017) 228-232.

[52] T. Li, Ideal strength and phonon instability in single-layer mos 2, Phys. Rev. B 85 (23) (2012) 235407.

[53] T. Lorenz, D. Teich, J.-O. Joswig, G. Seifert, Theoretical study of the mechanical behavior of individual tis 2 and mos 2 nanotubes, J. Phys. Chem. C 116 (21) (2012) 11714-11721.

[54] F. Liu, P. Ming, J. Li, Ab initio calculation of ideal strength and phonon instability of graphene under tension, Phys. Rev. B 76 (6) (2007) 064120.

[55] K. N. Kudin, G. E. Scuseria, B. I. Yakobson, C 2 f, bn, and c nanoshell elasticity from ab initio computations, Phys. Rev. B 64 (23) (2001) 235406.

[56] R. M. Martin, Electronic structure: basic theory and practical methods, Cambridge university press, 2004

[57] B. Mohan, A. Kumar, P. K. Ahluwalia, A first principle study of interband transitions and electron energy loss in mono and bilayer graphene: Effect of external electric field, Physica E 44 (7-8) (2012) 1670-1674.

[58] N. Wang, D. Cao, J. Wang, P. Liang, X. Chen, H. Shu, Interface effect on electronic and optical properties of antimonene/gaas van der waals heterostructures, J. Mater. Chem. C 5 (37) (2017) 9687-9693. 